\title{
Form Analysis by Neural Classification of Cells
}

\author{
Y. Belaïd and A. Belaïd \\ LORIA-CNRS \\ Campus scientifique B.P. 239 \\ 54506 Vandœuvre-lès-Nancy CEDEX France \\ \{ybelaid, abelaid\}@loria.fr
}

\begin{abstract}
Our aim in this paper is to present a generic approach for linearly combining multi neural classifier for cell analysis of forms. This approach can be applied in a preprocessing step in order to highlight the different kind of information filled in the form and to determine the appropriate treatment. Features used for the classification are relative to the text orientation and to its character morphology. Eight classes are extracted among numeric, alphabetic, vertical, horizontal, capitals, etc. Classifiers are multi-layered perceptrons considering firstly global features and refining the classification at each step by looking for more precise features. The recognition rate of the classifiers for 3. 500 cells issued from 19 forms is about $91 \%$.
\end{abstract}

\section{Introduction}

Form analysis becomes with the success of OCR/ICR techniques a very promising domain with different issues and applications. Several administrations and companies are today faced to a fast treatment of their forms in different domains such as order lecture, revenue form capture or multiple choice question paper analysis. Systems designed this last decade for form analysis are numerous and themes are varied. However, all of these systems are oriented towards a full form recognition without a real separation between the different phases. This makes difficult the reuse of systems and leads sometimes, for a new application, to the complete rewriting of the techniques. So, we have considered that for some classes of forms such as the tax forms, cells are the base of the form analysis and cell classification can constitute a generic part of a form analysis system.

Considering cell detection and extraction, the literature mentions mainly two approaches. The most common one deals with known forms and uses a detailed model for each class of forms $[2,3,4,8]$. Although the systems are efficient on specific forms, they can be hardly applied to other kind of forms. In opposite, the systems, in the other approach, ignore any a priori knowledge on the form and base the analysis mainly on cell analysis [5,6]. Although they are more general than the first ones and can be applied on a wide range of forms, their performance is limited because of their lack of knowledge.

Our aim in this paper is to propose an intermediate solution for unknown form analysis based on cell classification. Cells are first extracted from the form 
and classified according to different criteria based more on the content aspect than on its semantic interpretation.

The cell extraction and classification is a very important step in a form analysis process for several reasons:

- The information contained in the form is mainly located in the cells.

- The cell extraction allows to locate the information and to situate it according to the rows and columns. This leads to find at the same time the layout and the logical structure of the form (correspondence between rows and columns, number of dimensions, etc.).

- The exam of the content of each cell can help to the content classification, first by separating cells containing the information from empty ones, second, by analyzing the type of content (text, digits, etc.) in order to apply on it the adequate treatment.

- At last, the cell extraction and content classification can help to the preclassification of forms with a modest investigation.

The outline of this paper is as follows. After a brief description of the approach used for the cell location in section 2, we present in section 3 the different classes retained for the classification and give in section 4 the classification schema. Details dealing with the main classification steps will be then exposed in this section. So, we show the different features used for the different classes and the hierarchy of neural architectures. At last, before concluding, some experiments and results will be discussed in section 5 .

\section{Cell Extraction}

As mentioned in [7] cell location and extraction is operated in three steps.

In the first step, lines are detected in the image by applying Hough Transform. This technique was used for its robustness and reliability. It transforms the following line problem in a counting point problem [1]. In order to avoid a multitude of line candidates, voting points are limited to only those belonging either to the contours or to the black or gray areas. A recursive cut of the polar parameter space of lines and a fusion of close cells allow to fast locate the accumulation areas.

In the second step, segments associated to the lines, are extracted from the image. The line following is operated by advantaging the closest black pixels of the Hough lines. The lines detected can be simple, double, continuous or discontinuous, contours of black and gray areas, or vertical alignments of parentheses.

The cells are located at the third step. They are represented by a graph which arcs are the horizontal and vertical segments and which nodes are the intersection points between horizontal and vertical lines. Cells are given by the research for minimum circuits of the graph.

This first part of the system has been tested with success on French tax forms as well as on tables. The line extraction takes about 30" per image. 


\section{Cell Classes}

A detailed study of French tax forms leads us to define eight classes for cells described below:

- DIGI: it regroups the set of cells containing only digits. These digits generally correspond to amounts and can be preceded by the sign '+' or '-'.

- GRAY: it corresponds to gray areas which cannot be filled by any kind of data.

- HLET (for horizontal letters): all cells which text is horizontally aligned and which are constituted by alphanumeric chains containing lower-case letters and probably higher-case letters are affected to this class. They correspond essentially to form wordings.

- VLET (for vertical letters): it reassembles the same kind of cells within the class HLET but with text vertically aligned.

- HHCL (for horizontal higher-case letters): cells containing higher-case letters horizontally aligned and some digits or symbols such as parentheses are attributed to this class. These cells often correspond to wordings representing amounts.

- VHCL (for vertical higher-case letters): it corresponds to the class HHCL but considering only cells which text is vertically aligned.

- BLAC: it regroups cells in inverse video (with black foreground). These cells play a particular role in our forms.

- EMPT (for empty classes): this class regroups all the cells containing any data.

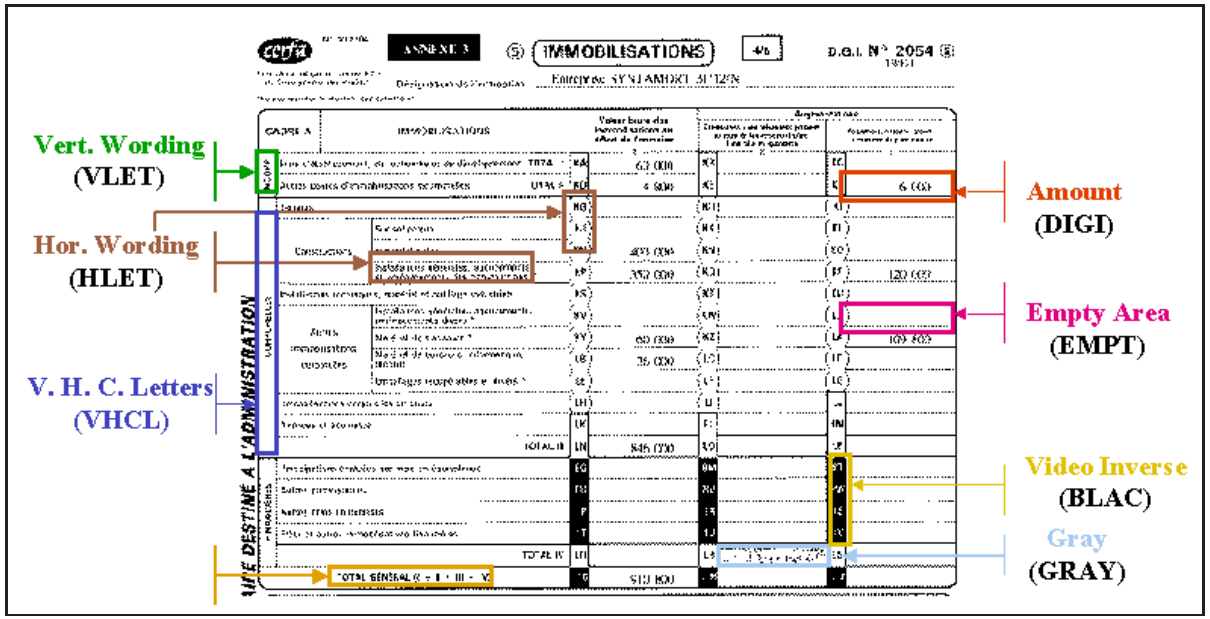

Fig. 1. Example of Classes. 
The choice of these classes depends of course on our application but can be adapted on other kind of forms. Figure 1 gives an example of a form highlighting these different classes.

\section{Analysis Phases}

The analysis schema consists of three main phases (cf. fig. 2:

- In the first phase, cells are extracted by line searching, segment reconstruction and cycle delimitation within the graph of line crossings

- In the second phase, some features are extracted from the cells either globally on the hole data or more precisely on the different connected components within each cell

- In the third phase, these features are analyzed by a list of classifiers which decide for one belonging class among the eight classes defined below
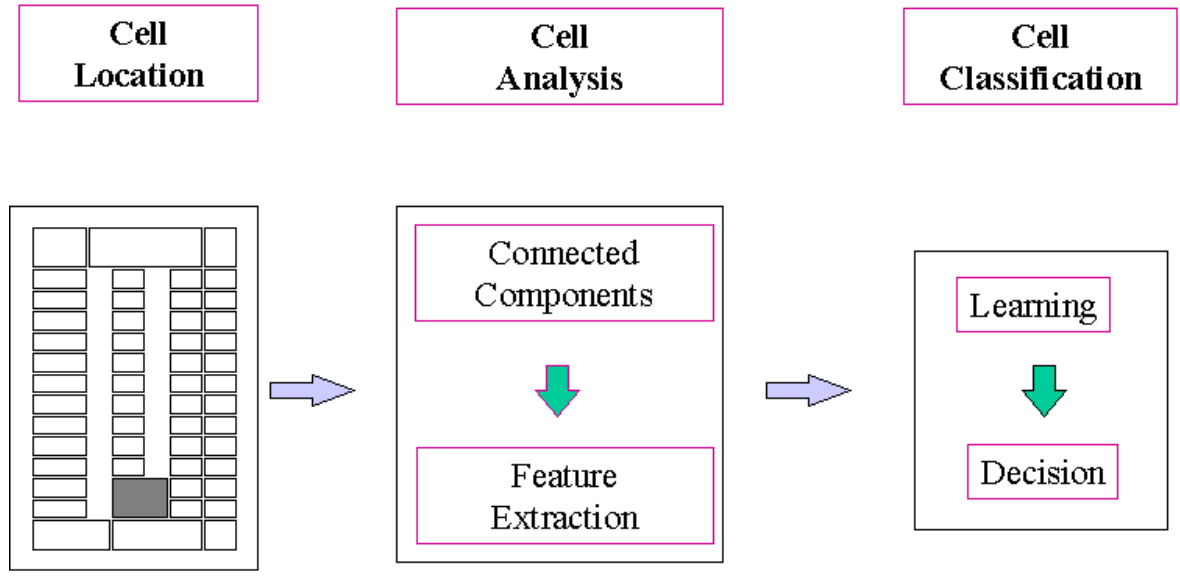

Fig. 2. Analysis Phases.

\subsection{Cell Extraction}

For line extraction, we used Hough transform on polar coordinates. Because of its slowness, the original method was improved by a filtering of voting points and a clever clustering of the accumulator cells in the Hough space (depending on line direction). 
For filtering, only points belonging to contours vote. Thanks to this filtering, the system is not disturbed by the black strips and can detect accurately their contour. For thick lines, the two edges are merged.

The clustering in the polar coordinates is made up by a recursive cutting up algorithm.

Figure 3.a shows the hough straight line, the voting pixel and the current segment followed. Figure 3.b shows the line extraction by Hough. We can notice that lines made up by brackets and black strips are also found. Finally, Figure 3.c synthesises the different extraction rates, for well delimitation, under- and oversegmentation, and for omission.

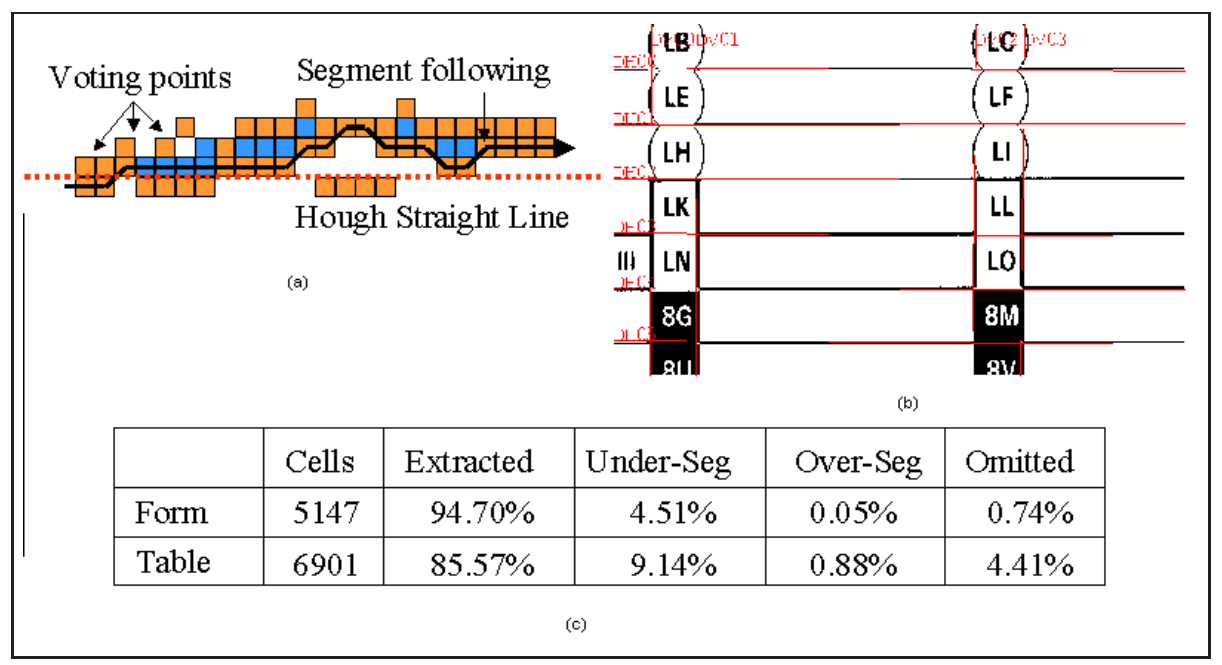

Fig. 3. Cell Extraction.

The method tolerates a reasonable orientation. Each item is depicted by a box in a specific colour. Even though the method gives a very indifferent quality, it provides a good base for a global classification of the cells. Joined characters and overlapping characters with horizontal lines are usually disturbing the classification(cf. fig. 4.

\subsection{Cell Classification}

The cell classification schema can be divided into three steps as shown if fig. 5 .

The classification is performed progressively in order to discard rapidly the obvious cases and to refine the others.

The role of the first step is to give a rapid decision on the simple cases such as GRAY, EMPT and BLAC and to separate the remaining classes into two main 


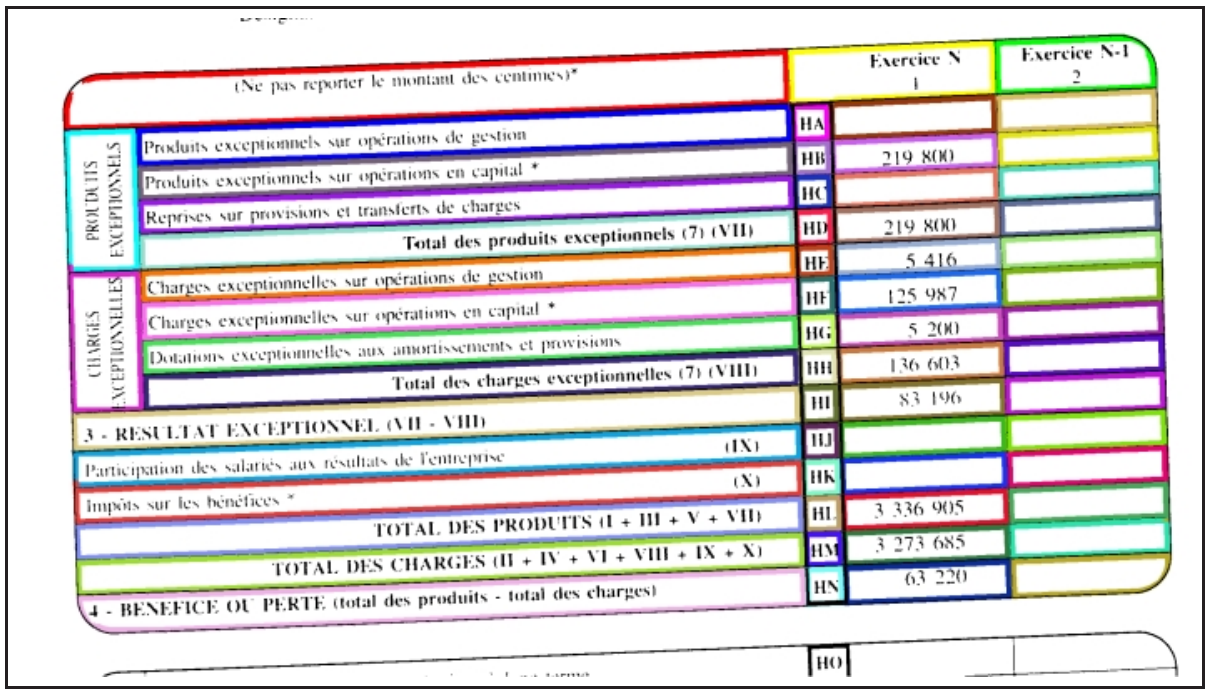

Fig. 4. Cell Extraction.

categories depending on the alignment: horizontal or vertical. Because of the ill quality of the image, this classification is operated globally on the cell images by using some numeric features.

The objective of the second step is to refine the classification within both categories The classification is operated directly on the image of the CCs of the cell. Two specific neuronal nets are used at this level.

The third stage is used for some disturbing cases corresponding to cut characters such as the zero which seriously handicaps the straightforward classification of the digits. A specific classifier is used for a possible merging of cut digits.

\subsection{Classification from CC Parameters}

12 simple numerical parameters have been experimentally determined for the classification in the first step.

1. Number of connected components: this is related to the number of CCs after a merging step introduced because of the presence of numerous cut characters (cf. fig. 6). The merging is made within a cell, line by line. Two CCs are merged if they respect the following constraints:

- they belong to the same text line,

- they are consecutive in the line,

- they are superposed (cf. fig. 7.a) or overlapped with an important intersection area (cf. fig. 7.b) or overlapped with a small intersection and 


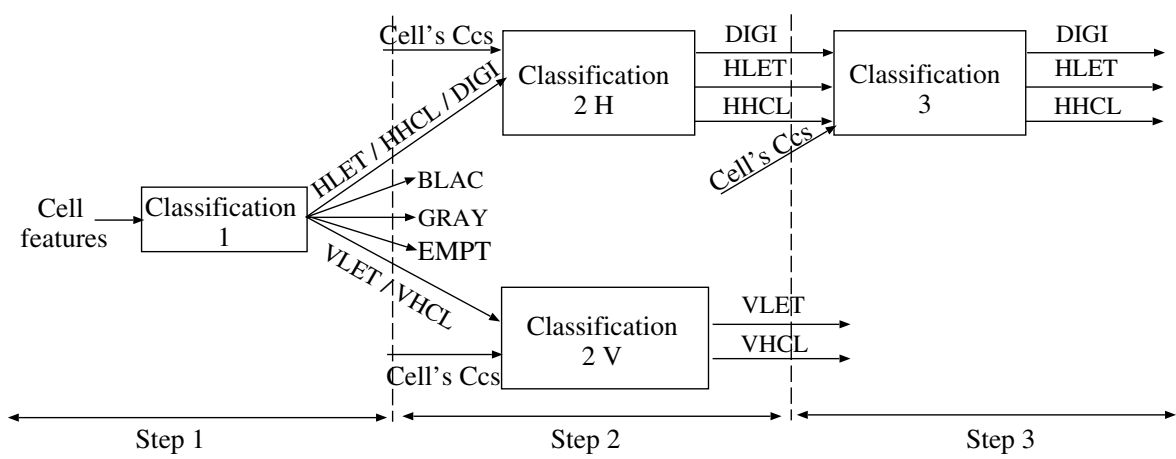

Fig. 5. Cell Classification.

\section{6}

Fig. 6. Connected Components Before Merging.

where one of the $\mathrm{CC}$ is very small compared to the average size of the Cell's CCs studied.

2. Text alignment: we have observed in the forms studied that the cells which are more wide than tall, contain text horizontally aligned. In opposite, when the cells are more tall then wide, the text can be aligned horizontally or vertically. An analysis of the text is then necessary in this case. Three cases are considered:

- the number of horizontal CCs, i.e. those which the height is greater than the width,

- the homogeneity of the height of the text lines,

- the height of the greater CCs of each line.

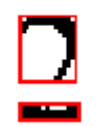

a) Merging of $2 \mathrm{CCs}$

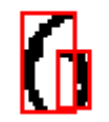

b) Overlapping of $2 \mathrm{CCs}$

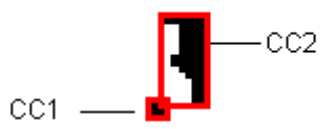

c) Size of $\mathrm{CC} 1$ is very small compared of the size of $\mathrm{CC} 2$

Fig. 7. Different configurations of Connected Components. 
If the number of horizontal CCs is important, text lines are homogeneous and if there are CCs in each line which height is similar to the line height, the text is considered as horizontally aligned. Otherwise, it is considered as vertically aligned.

3. Number of text lines: the text lines are detected by analyzing the histogram obtained by horizontal or vertical projection of the image.

This analysis is performed in three steps:

- In the first step, the black areas of the histogram are delimited. When the text is of a good quality and lines are not overlapped, each black area corresponds to a text line. To avoid to take into account the noise, only black areas are considered with a size experimentally fixed to 3 pixels for the height and 5 pixels for the width. It is important to combine the height and the width in order to avoid to consider as noise, lines containing only one character (cf. fig 8).

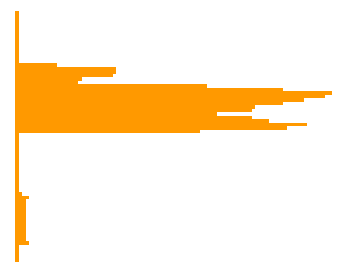

\section{Exercice $\mathbf{N}$ \\ 1}

Fig. 8. Example of a Line containing only one Character.

- In the second step, picks found are merged where they are close (separated with less than 3 pixels). In fact, in some cases, a line can be represented by two picks. This is the case for the line of 9 because the letter 'g' of the word 'outillage' is composed of two CCs.

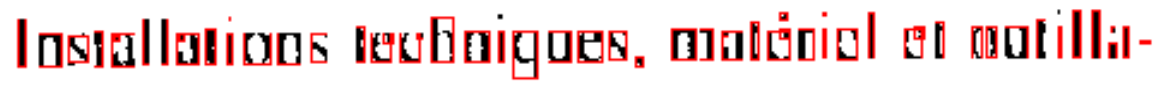 gè industrists}

Fig. 9. Bad Segmented Characters.

- Lines previously found are examined in order to separate couples of consecutive lines connected by the down-strokes of the ones or the stems of the others (cf. fig 10). For this, each black area is analyzed so that picks separated by a valley which height is less than a threshold 's' which value 
has been fixed at $28 \%$ of the greatest height (cf. fig 11) are extracted. These picks are compared if they are neghbours. We begin by analyzing the picks having the deepest valleys. If the distance between a pair of picks is less than the sum of the width of the two picks, this probably indicates the presence of two close text lines. If the widths of these two potential lines are comparable, then two separated lines are considered. In the other cases, we merge the two picks which forms a single line. When all the picks have been treated by pairs, we compare the size of the lines obtained during this step. If their size is homogeneous, the lines are selected, else only lines extracted in the second step are preserved.

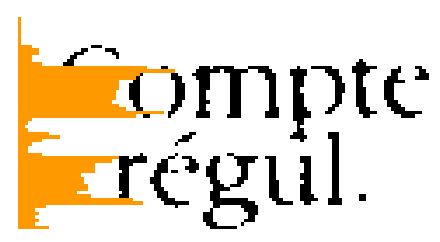

Fig. 10. Very Close Text Lines.

4. Number of classes of CC heights. It is obtained from the analysis of height histogram of the CCs.

A pick is indicated by a high value of the histogram. The class searching is determined as follows:

\section{Begin}

Create a class with the biggest pick.

Examine the others picks in a decreasing order.

Let $\mathrm{Pc}$ be the current pick, search $\mathrm{Pg}$ a bigger pick than $\mathrm{Pc}$ and the closest of Pc.

If $\mathrm{Pc}$ is enough close to $\mathrm{Pg}$ then Pc belongs to the same class than $\mathrm{Pg}$

\section{End} else create a new class containing Pc.

5. Number of CC width classes.

6. Number of width classes of the spaces between CCs.

7. Average number of black segments per line in a $\mathrm{CC}$.

8. Average density of black pixels per $\mathrm{CC}$.

9. Density of black pixels in a cell.

10. Average height of CCs.

11. Number of CCs deleted: this value is determined during the CC extraction step; it corresponds to the number of CCs assimilated to the noise.

12. Ratio between the number of CCs deleted and the total number of CCs. The choice of these 12 parameters results from a series of observations and tests realized on a database built up for this problem and from which we have 


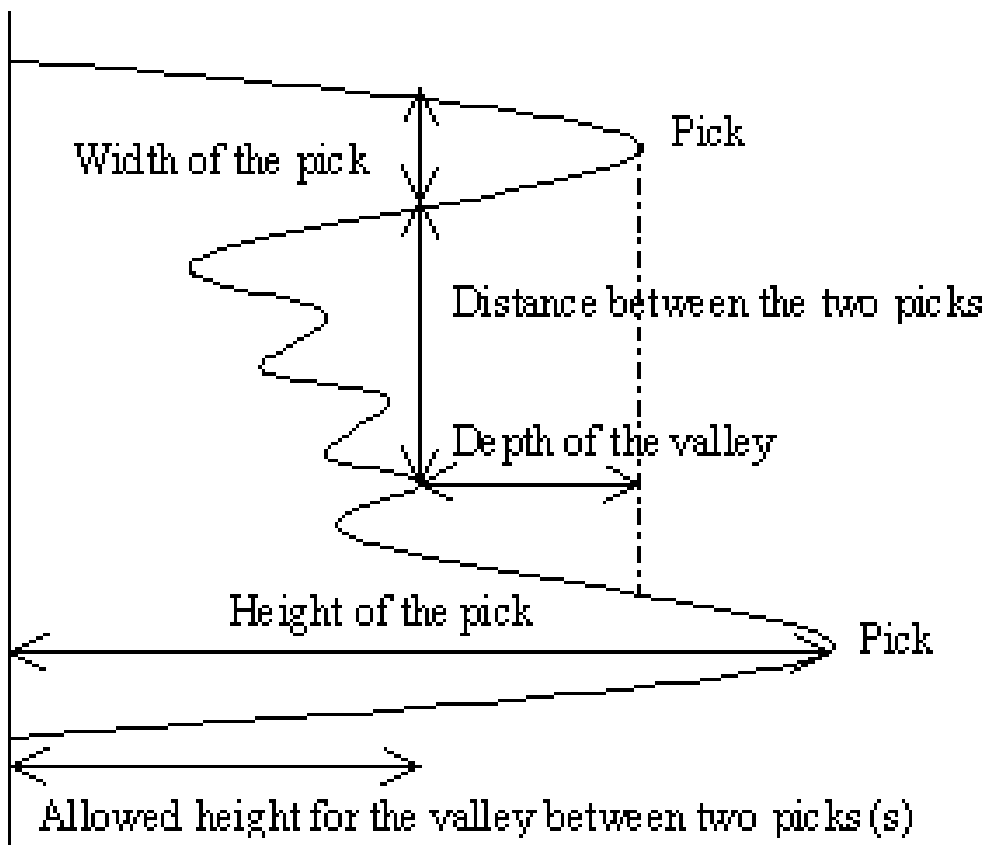

Fig. 11. Example of two Consecutive Picks Used for Line Detection.

verified the contribution of each one of these parameters in the classification process.

The classifier uses a mono-layer perceptron with 12 neurons on the entry layer (for the 12 parameters) and 5 neurons on the exit layer (for the 5 classes retained).

\subsection{Classification from Cell CCs}

This phase including the steps 2 and 3 of the classification process, tends to classify cells containing text into three classes: DIGI, HLET and HHCL for cells with horizontal alignment or into two classes: VLET and VHCL for cells with vertical alignment. In the tax forms, there was not amounts vertically aligned. This explains the class difference in relation with the alignment.

In the second step, the entry data of the classifier is the size normalized image of a CC. The classifier retained is a perceptron with a hidden layer. It contains 64 neurons on the entry layer (the 64 pixels of the normalized image of a CC), 12 neurons on the hidden layer and 2 or 3 neurons on the output layer according to the alignment of the studied cell. A value is associated to each output. For every output, we compute the product of the values of each $\mathrm{CC}$ of the considered cell. The output having the higher product is attributed to the cell. 
The results obtained at the end of this step are satisfactory except for the DIGI class. Errors come essentially from the '0' often bad segmented and cut in two parts. So, cells containing a majority of ' 0 ' are bad classified. The solution for this problem is given in the third step.

In this step, CCs presented to the classifier are normalized and grouped by pairs. This treatment is realized for cells which number of height class of CCs is 1 or 2. The classifier used is a perceptron having 128 neurons on the entry layer (the 128 pixels of the image normalized and merged into $2 \mathrm{CCs}$ ), 18 neurons on the hidden layer and 2 neurons on the output layer: one for the ' 0 ' class and one for the other characters.

These results are compared with those of the step 2 and a decision is taken for the belonging or not of the cell to the classes DIGI, HLET or HHCL.

A score is determined for each one of the three classes and we retain the one which score is the highest. Let notice

ScDIGI: the score of the class DIGI for the cell considered,

ScHLET: the one of HLET,

ScHHCL: the one of HHCL,

$\mathrm{SjC} 2 \mathrm{Hcci}$ : the output value $\mathrm{j}$ of the classifier $2 \mathrm{H}$ for the $\mathrm{CC} \mathrm{i}$,

$\mathrm{SjC} 3 \mathrm{cci}$ : the output value $\mathrm{j}$ of the classifier 3 for the $\mathrm{CC}$ i merged with the $\mathrm{CC}$ $\mathrm{i}+1$.

The computations are made as follows:

\section{Begin}

$$
\text { ScDIGI, ScHLET, ScHHCL }=1
$$

$\mathrm{i}=1$

For every CC i of the current cell do

If $\mathrm{S} 1 \mathrm{C} 3 \mathrm{cc}$ i i $\mathrm{S} 2 \mathrm{C} 3 \mathrm{cc}$ i then

$\mathrm{ScDIGI}=\mathrm{ScDIGI} * \mathrm{~S} 1 \mathrm{C} 2 \mathrm{Hcci}$

$\mathrm{ScHLET}=\mathrm{ScHLET} * \mathrm{~S} 2 \mathrm{C} 2 \mathrm{Hcci}$

$\mathrm{ScHHCL}=\mathrm{ScHHCL} * \mathrm{~S} 3 \mathrm{C} 2 \mathrm{Hcci}$

$\mathrm{i}=\mathrm{i}+1$

Else

$$
\begin{aligned}
& \text { ScDIGI }=\text { ScDIGI } * \text { S1C3cci } \\
& \text { ScHLET }=\text { ScHLET } * \text { S2C2Hcci } * \text { S2C2Hcci }+1 \\
& \text { ScHHCL }=\text { ScHHCL } * \text { S2C2Hcci } * \text { S2C } 2 \text { Hcci }+1 \\
& \mathrm{i}=\mathrm{i}+2 \\
& \text { Endif }
\end{aligned}
$$

\section{Endfor}

\section{End}

\section{Results and Discussion}

The classification process was tested on 19 French tax forms belonging to the General Direction of French Revenue. The classification time for one form is about 1'45" on a SUN Ultra Spark station, model $140 \mathrm{MHz}$. 
The results obtained are detailed in the table 1 and the classification rates are presented in table 2. A classification example of a form is given in 12 . The color attributed to each cell indicates the class found (cf. fig 3). A classification error is materialized by the presence of a little square on the bottom left. It has the colour of the wanted class.

\begin{tabular}{|c|c|c|c|c|c|c|c|c|c|}
\hline & DIGI & HLET & HHCL & VLET & VHCL & GRAY & BLAC & EMPT & $\begin{array}{c}\text { Total } \\
\text { Number } \\
\text { of Cells }\end{array}$ \\
\hline DIGI & 638 & 9 & 6 & 0 & 0 & 0 & 0 & 0 & 653 \\
\hline HLET & 5 & 587 & 233 & 0 & 0 & 4 & 0 & 0 & 829 \\
\hline HHCL & 4 & 22 & 308 & 4 & 2 & 2 & 0 & 0 & 342 \\
\hline VLET & 0 & 0 & 0 & 23 & 0 & 0 & 0 & 0 & 23 \\
\hline VHCL & 0 & 0 & 0 & 0 & 72 & 0 & 0 & 0 & 72 \\
\hline GRAY & 0 & 0 & 1 & 0 & 0 & 23 & 1 & 0 & 25 \\
\hline BLAC & 0 & 0 & 0 & 0 & 0 & 0 & 72 & 0 & 72 \\
\hline EMPT & 0 & 0 & 2 & 0 & 0 & 7 & 0 & 1479 & 1488 \\
\hline
\end{tabular}

Table 1. Form Classification Results.

\begin{tabular}{|c|c|c|c|c|c|c|c|c|}
\hline DIGI & HLET & HHCL & VLET & VHCL & GRAY & BLAC & EMPT & TOTAL \\
\hline $97.70 \%$ & $70.81 \%$ & $90.06 \%$ & $100.00 \%$ & $100.00 \%$ & $92.00 \%$ & $100.00 \%$ & $99.40 \%$ & $91.38 \%$ \\
\hline
\end{tabular}

Table 2. Classification Rates.

We can remark that the scores are very good for the classes DIGI, VLET, VHCL, GRAY, BLAC and EMPT, but are less good for the classes HLET and HHCL. There are several reasons explaining the confusions:

- The bad quality of images can produce an over-segmentation (cf. fig. 11) or a under-segmentation (cf. fig. 12).

- Some characters have the same morphology in lower-case and higher-case and cannot be differentiated after normalization. It is the case of characters as 'c', 'o', 's', 'u', 'v', 'x' and 'z'.

Several solutions can be considered in order to resolve these problems:

- creation of a reject class for cells for which it is difficult to make a choice between the classes HLET and HHCL,

- fusion of the classes HLET and HHCL: this fusion gives a general classification rate equal to $98,32 \%$,

- the consideration of the CC height before normalization. 


\begin{tabular}{|c|c|c|c|c|c|c|c|}
\hline DIGI & HLET & HHCL & VLET & VHCL & GRAY & BLAC & EMPTY \\
red & brown & orange & clear green & dark green & cyan & yellow & mauve \\
\hline
\end{tabular}

Table 3. Colors of the eigh Cell Classes.

\section{Conclusion}

This paper outlines a feasibility study for the classification of form cells into eight classes depending on the presence of information or not, on the text alignment: horizontal or vertical and the character modes higher-case or lower-case. Few systems have been developed in this sense. Most of the classification methods developed try to differentiate between text and non text areas. We used a perceptron for the classification. It is mono-layer for the first step which realizes a pre-classification by using numerical parameters. It contains one hidden layer for the steps 2 and 3 which analyze the text areas from normalized images of CCs. The results obtained are acceptable. Improvements and adaptations remain are possible. Acknowledgements: The authors wish to thank N. Pican for providing us with his implementation of the perceptron algorithm.

\section{References}

1. RISSE T., Hough Transform for Line Recognition : Complexity of E vidence Accumulation and Cluster Detection, Computer Vision, Graphics, and Image Processing, Vol. 46, 1989, pp. 327-345. 59

2. CASEY, FERGUSON D., MOHIUDDIN K. and WALACH E., Intelligent Form s Processing System, Machine Vision and Applications, Vol. 5, no 3, 1992, pp. 14 4-155. 58

3. ISHITANI Y., Model Matching Based on Association Graph for Form Image Understanding, in Proceedings of ICDAR'95 : 3rd International Conference o n Document Analysis and Recognition, Montréal, Canada, 1995, pp. 287-292. 58

4. YUAN J., TANG Y. Y. and SUEN C. Y., Four Directional Adjacency G raphs (FDAG) and Their Application in Locating Fields in Forms, in Proceedings o $\mathrm{f}$ ICDAR'95 : 3rd International Conference on Document Analysis and Recognition, Montréal, Canada, 1995, pp. 752-755. 58

5. HIRAYAMA Y., Analysing Form Images by Using Line-Shared-Adjacent Cell Relations, in Proceedings of ICPR'96 : 13th International Conference on Pa ttern Recognition, 1996, pp. 768-772. 58

6. SHIMOTSUJI S. and ASANO M., Form Identification based on Cell St ructure, in Proceedings of ICPR'96 : 13th International Conference on Pattern Re cognition, 1996, pp. 793-797. 58

7. TUROLLA E. BELAïD Y. and BELAïD A., Form item extraction based on line searching, in Graphics Recognition : Methods and Applications, Lecture Not es in Computer Science, Vol. 1072, 1996, pp. 69-79. 59

8. ARAI H. et ODAKA K., From Processing Based on Background Region Analysis, in Proceedings of ICDAR'97 : 4th International Conference on Document Analysis and Recognition, Ulm, Allemagne, Vol. 1, 1997, pp. 164-169. 58 


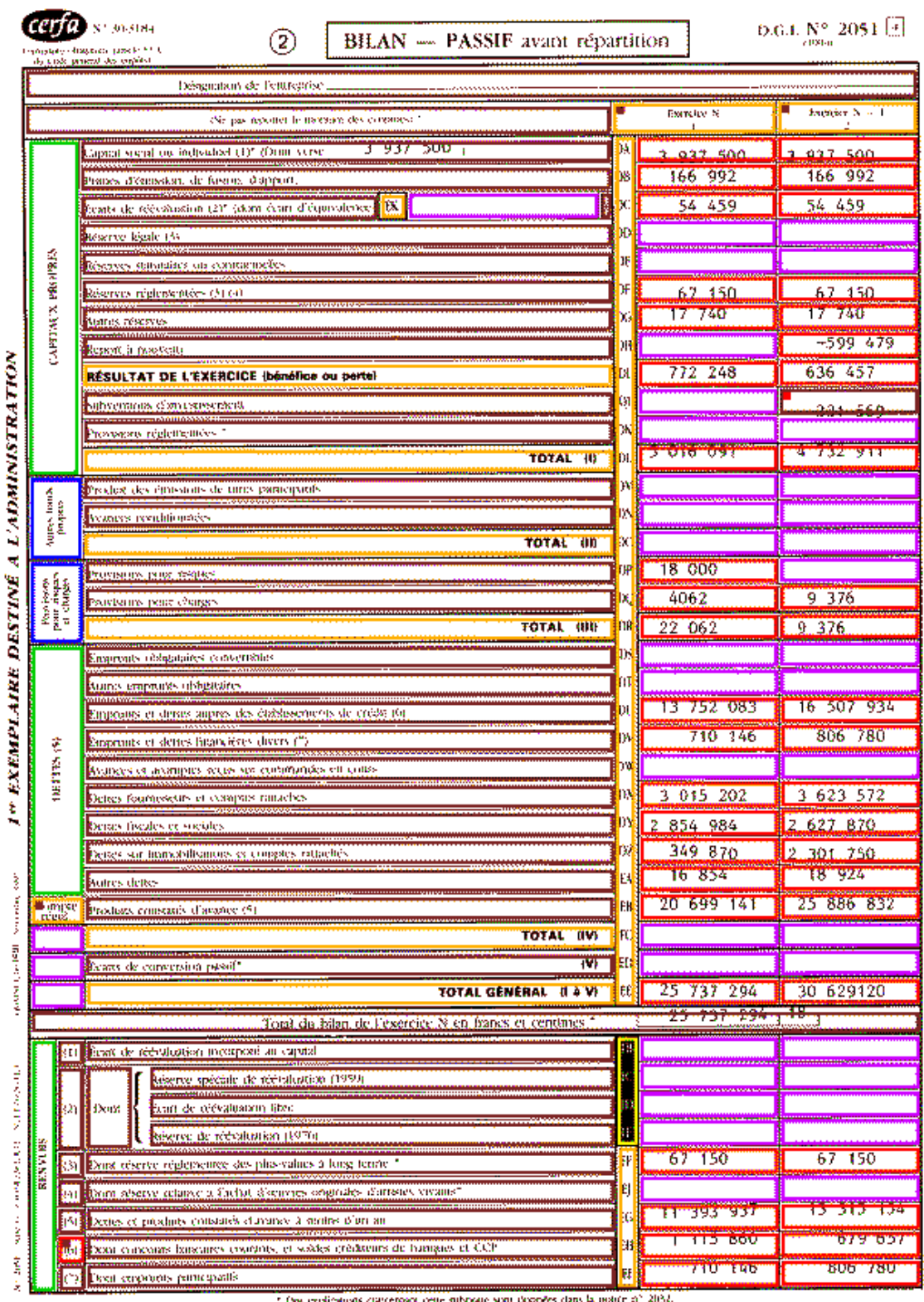

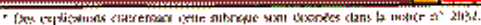

Fig. 12. An Example of Form Classification. 